\title{
DYNAMICS OF DISCLINATIONS IN LIQUID CRYSTALS
}

\author{
BY \\ L. M. PISMEN AND J. RUBINSTEIN \\ Technion-I.I.T., Haifa 32000, Israel
}

1. Introduction. It is widely recognized that topological defects play an important role in both statics and dynamics of liquid crystals. By defects one usually refers to singularities in the order parameter space. A somewhat wider definition, which is needed in liquid crystal theory, will be given below. The statics of defects has been extensively studied, though open questions abide. A recent comprehensive review of the field was given by Kleman [8]. A deep and beautiful topological theory [9] governs the classification of stable defects, placed in a more general context of nonlinear field theories. Topological considerations need, however, to be complemented by energetics. This presents major difficulties. Considerable advances were recently made through the introduction of new theoretical and numerical tools $[2,6]$.

The dynamics of defects in liquid crystals is still not well known. The problem of deriving equations of motion of field singularities is one of the most complex problems in nonlinear field theories. This problem has been addressed in particular in the phenomenological theory of superfluidity [10], and in the theory of nonequilibrium patterns $[15,3,1,11]$. The latter bears perhaps the closest connection to liquid crystals problems. It encounters similar difficulties that largely stem from the fact that the total energy of point defects in $2 \mathrm{D}$ or line defects in $3 \mathrm{D}$ diverges with the size of the system.

The method of matched asymptotic expansions has been recently applied successfully $[10,11,16]$ to study the motion of defects in dissipative systems and superfluids. We shall apply it here to three different (but topologically equivalent) models in the liquid crystal theory. The first case is line defects (disclinations) in nematics that are stabilized by an external field. The second problem, also in the context of line defects in nematics, is associated with a recent model due to Ericksen [4]. Ericksen's model includes the effect of variable degree of orientation on the energy of the director field. Finally, we shall consider the dynamics of disclinations in smectic $\mathrm{C}$ liquid crystals. We shall show that a similar law of motion for the disclinations holds for all these cases.

In the next section we shall present the basic equations governing models of this kind and give some static solutions. Far field equations governing the behavior away from the core of the disclinations will be derived and solved in Sec. 3. In Sec. 4 we work out the structure of the core. Matching the core and far field expansions, we Received January 15, 1991 and, in revised form, April 1, 1991.

(C)1992 Brown University 
obtain the equations of motion of the defects under an arbitrary weak external field. This field could, in particular, be induced by other defects. Thus we can also study the interaction of several defects.

2. Basic equations. All the problems we consider here are associated with a Lagrangian of the form

$$
\left.G(u)=\frac{1}{2} \int \mid g_{a b} \nabla u^{a} \nabla u^{b}+V(u ; H)\right] d^{d} \mathbf{x},
$$

where $u: R^{d} \times[0, T] \rightarrow R^{p}$ is the order parameter function, $H$ refers to an external field and $g$ is a metric tensor of the order parameter space. We shall adopt the generalized notion of defect introduced by us in [13]. For this purpose we consider the manifolds $V=$ const in the order parameter space and call them strata. A smooth parametrization of the strata will be called phase variables. Of particular importance here are minimal strata, which are the strata on which $V$ achieves a local minimum. Dissipative systems evolve towards the minimal strata. Topological considerations, however, might prohibit a global parametrization by phase variables restricted to the minimal strata.

By codimension $n$ defect we mean a $d-n$ manifold in the $d$-dimensional physical (real) space where the actual solution cannot be described by the phase variables of a minimal stratum alone. In this paper we consider codimension 2 defects, i.e., point defects in the plane or line defects in space. In the liquid crystals theory such defects are often called disclinations. Throughout the paper we shall use the words disclination, defect, and vortex as synonyms.

Liquid crystals are characterized by a macroscopic director field. This field represents the preferred orientation of the molecules. Various liquids may differ in the way in which the director components interact with each other and with additional internal and external parameters. We assume that the dynamics is dominated by the elastic energy of the liquid crystal, i.e., we neglect the fluid dynamical part. Thus the order parameter $u$ evolves according to

$$
\partial_{t} u=-g^{a b} \frac{\delta G}{\delta u^{b}} .
$$

In addition we shall restrict ourselves to the one constant approximation in the Frank energy [5]. The following three models can be analysed within a single framework.

1. Nematics in external fields. An external field $\vec{E}$ acts on the macroscopic director field, presented by the unit vector $\hat{u}$ in $R^{3}$. The orientation is presumed perfect; thus, the order parameter space is the surface of the unit sphere, and can be parametrized by two angles $\alpha, \beta$. Hence, the appropriate metric tensor in (1) is

$$
g_{a b}=\operatorname{diag}\left(1, \sin ^{2} \alpha\right) .
$$

We are interested in the case where the director tends to be oriented across the field $\vec{E}$. Such a perpendicular orientation is common in nematics under an electrical field, though it is rare when the imposed field is magnetic [5]. The potential $V=\vec{E} \cdot \hat{u}$ breaks the spherical symmetry of the order parameter space. The residual symmetry 
of the strata $V=$ const is the symmetry to rotation around the direction of the external field $\vec{E}$.

Assuming that the polar axis is oriented along the external field, and scaling the coordinates so that the field strength is normalized to unity, the potential $V$ is given by

$$
V=1-\sin ^{2} \alpha .
$$

The corresponding evolution equations, which are the gradient flow of the Lagrangian (1), are

$$
\begin{aligned}
& \partial_{t} \alpha=\nabla^{2} \alpha-\frac{1}{2} \sin 2 \alpha\left(|\nabla \beta|^{2}-1\right), \\
& \partial_{t} \beta=\nabla^{2} \beta+2 \cot \alpha \nabla \alpha \cdot \nabla \beta .
\end{aligned}
$$

The system (5), (6) has stationary circularly symmetric solutions corresponding to quiescent line vortices with an integer topological charge (degree) $n$ oriented along the imposed field. Stable defects have the unit charge $n= \pm 1$. The solutions have the form

with $\alpha_{0}(r)$ satisfying

$$
\beta=n \phi, \quad \alpha=\alpha_{0}(r),
$$

$$
\alpha_{0}^{\prime \prime}+r^{-1} \alpha_{0}^{\prime}+\frac{1}{2}\left(1-n^{2} r^{-2}\right) \sin 2 \alpha_{0}=0 .
$$

Here $(r, \phi)$ are polar coordinates in the plane. Equation (8) is solved with the boundary conditions

$$
\alpha_{0}(0)=0, \quad \alpha_{0}(\infty)=\frac{\pi}{2} .
$$

The first condition insures regularity at the origin. The second condition implies that the director is favorably oriented far from the vortex core.

It is instructive to see how the presence of the external field changes in a qualitative way the character of the vortex solution. When the field is absent, there is a continuous family of nontrivial solutions of the form $\alpha_{0}=2 \arctan (r / l)^{n}$, that satisfy

$$
\alpha_{0}^{\prime \prime}+r^{-1} \alpha_{0}^{\prime}-\frac{1}{2} n^{2} r^{-2} \sin 2 \alpha_{0}=0 .
$$

Here $l$ is an arbitrary constant measuring the extent of the vortex core. As the core shrinks or expands adiabatically when $l$, respectively, increases or decreases, the vortex in the unforced system dissipates into a trivial solution $\left(\alpha_{0}=0\right.$, or $\left.\alpha_{0}=\pi\right)$. Physically this corresponds to the removal of the singularity by "escape in third dimension" [9].

The solution of (8), on the contrary, is unique. Due to the extra forcing term, the stationary point $\alpha_{0}=\pi / 2$ becomes a saddle as $r \rightarrow \infty$ when (8) is viewed as a dynamical system in $r$. There exists a single trajectory approaching this point as $r$ increases. Near the origin the polar angle increases like $A r^{|n|}$. The indefinite constant $A$ has to be adjusted so that the trajectory approaches the saddle point. There is a unique value of $A$ insuring the convergence. For $n=1$, the computation yields $A=1.1922144$. The solution obtained by numerical integration ${ }^{1}$ of $(8)$ for

\footnotetext{
${ }^{1}$ We are thankful to $J$. D. Rodriguez for carrying out this computation for us.
} 


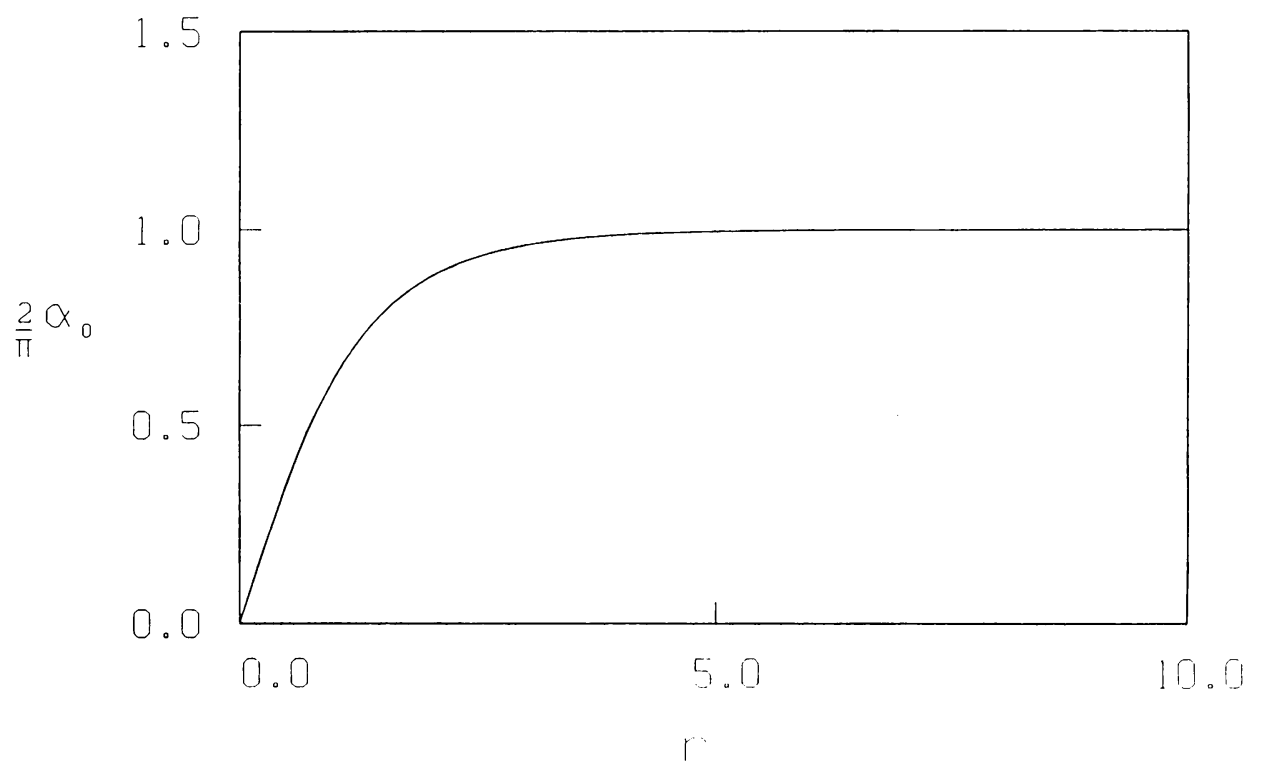

Fig. 1. The radial dependence of the polar angle in quiescent vortex $(n=1)$.

$n=1$ is shown in Fig. 1. The limiting value $\alpha_{0}=\pi / 2$ is approached exponentially fast as $r \rightarrow \infty$.

2. Smectic $C$ liquid crystals. Smectics are liquid crystals with a layered structure. We denote by $\hat{p}$ the unit normal to the layers, and by $\alpha$ the angle between the director $\hat{u}$ and $\hat{p}$. The elastic energy depends on the projection $s=|\hat{u} \times \hat{p}|=\sin \alpha$. In smectic $C$ liquids the energy is at a minimum at a certain angle $\alpha_{0}$ differing from 0 and $\pi / 2$. In smectics $C^{*}$ there is, in addition, a twist of the director across the layers. In thin films the difference between both cases is negligible.

As in the preceding subsection, the symmetry of the order parameter space is broken, and the residual symmetry is that of the rotations of the unit vector $\hat{v}$ in the layer plane. We shall write the energy functional for a flat layer of constant thickness neglecting the distinction between energies of the splay and bend deformations but keeping a separate elastic constant $k$ corresponding to spatial changes of $s$ :

$$
\begin{gathered}
G(u)=\frac{1}{2} \int\left[s^{2}|\nabla \hat{v}|^{2}+k|\nabla s|^{2}+V(s)\right] d^{2} x, \\
V(s)=\frac{1}{2 c^{2}} s^{4}-s^{2} .
\end{gathered}
$$

Parametrizing $\hat{v}$ by the polar angle $\beta$ we write the evolution equations (assuming $k=$ const) in the form

$$
\begin{aligned}
\partial_{t} s & =k \nabla^{2} s-|\nabla \beta|^{2} s-V^{\prime}(s), \\
\partial_{t} \beta & =\nabla^{2} \beta-2 s^{-1} \nabla s \cdot \nabla \beta .
\end{aligned}
$$

Rather than $s$, one can take as an order parameter the angle $\alpha$ or, for that matter, any function of both. The alternatives differ by the choice of the metric, and the most 
advantageous choice is that incorporating the dependence of $k$ on the parameter into the metric factor.

As in the case considered above, there is a stationary defect of the form

$$
v=s \hat{v}, \quad \hat{v}=(\cos \beta, \sin \beta), \quad \beta=n \phi, \quad s=s_{0}(r),
$$

where $n$ is integer and $s_{0}(r)$ solves

$$
\frac{k}{r}\left(r s_{0}^{\prime}\right)^{\prime}-\frac{n^{2}}{r^{2}} s_{0}-V^{\prime}\left(s_{0}\right)=0, \quad s_{0}(0)=0, \quad s_{0}(\infty)=c .
$$

The solution of this equation is similar to that of eq. (8). Again, at $r \rightarrow 0 s_{0}=A r^{|n| / k}$ with a constant $A$ to be adjusted in the course of numerical integration. The constant $c$ in the potential can be eliminated by rescaling $s_{0} \rightarrow c s_{0}$. Then eq. (16) is identical at $k=1$ to that defining the structure of the vortex in the Ginzburg-Landau equation.

3. Nematics with variable degree of orientation. In the classical theory of liquid crystals [5] the director field is confined everywhere to the unit sphere $S^{2}$. Topological considerations indicate that such a description might break down at some points or lines (the defects), where the director will not be defined at all. Physically, this can be seen as local "melting" of the liquid crystal into an isotropic fluid. This does not happen on line singularities with an integer charge due to the possibility of the "escape in third dimension" that renders integer charge line singularities in unforced nematics topologically unstable. In the presence of an orienting field, the same "escape" phenomenon allows the relaxation of stresses without local melting, as described in the preceding subsection. Near point defects in $R^{3}$, melting is unavoidable but is not important energetically, since the energy of the director field does not diverge in this case as $r \rightarrow 0$. The most interesting case, where melting is both unavoidable and necessary to prevent divergencies in the field energy, is the line singularity with a half-integer charge, or, restricting to the stable case, $n= \pm 1 / 2$.

To retain the physical meaning of the director near the defects, Ericksen proposed [4] a modified model incorporating another component of the order parameter-the degree of orientation $s(x, t)$ measuring the extent to which the microscopic alignment agrees with the macroscopic director. The degree of orientation appears also in the classical theory [5], particularly, when the phase transition between nematic and isotropic phases is considered, but spatial changes of $s$ in conjunction with the changes or the orientation of the director are not incorporated there explicitly.

The degree of orientation $s$ in Ericksen's theory takes values in the interval $\left[-\frac{1}{2}, 1\right]$. Negative values, corresponding to preferred orientations normal to the director, are not realized physically. We shall consider therefore only $s$ lying within the interval $[0,1]$. The order parameter space is now the interior of the unit ball. The metric tensor can be taken in the form

$$
g_{a b}=\operatorname{diag}\left(1, s^{2}, s^{2} \sin ^{2} \alpha\right) .
$$

The energy functional can be written as

$$
G(u)=\frac{1}{2} \int\left[s^{2}|\nabla \hat{u}|^{2}+k|\nabla s|^{2}+V(s)\right] d^{3} x,
$$


where $k$ is the dimensionless elastic constant accounting for contributions of spatial variations of $s$. The energy associated with rotations of the director is written in the one-constant approximation. We shall assume the quartic potential

$$
V(s)=2 \int s(s-b)(s-c) d s
$$

that corresponds to equilibria at $s=0, s=b$, and $s=c$. The physically relevant case is $0<b<c / 2$ when both the isotropic $(s=0)$ and nematic $(s=c)$ phases correspond to minima of the potential but the isotropic phase is metastable.

At a defect, we expect the nematic to melt locally, so that $s=0$, and the macroscopic director becomes meaningless. The Euler-Lagrange equations of the functional (18) have a stationary vortex line solution with the director $\hat{u}$ lying everywhere in the $(x, y)$ plane:

$$
\hat{u}=(\cos \alpha, \sin \alpha \cos \beta, \sin \alpha \sin \beta), \quad \alpha=\frac{\pi}{2}, \quad \beta=n \phi, \quad s=s_{0}(r),
$$

with $s_{0}(r)$ solving eq. (16). Restricting to the "planar" case $\alpha=\pi / 2$, we can take as the order parameter space the interior of the unit circle. Then the evolution equations for $(s, \beta)$ are of the same form as (13), (14).

3. Far field. The equations in the preceding section are supposed to be dimensionless. We introduce now a small parameter $\varepsilon$. This parameter measures the small variation in the field in which the defect is immersed. It could be, for instance, the reciprocal of the nondimensional distance between two or more disclinations. The analysis is essentially the same for all three cases. Therefore we discuss in detail the first case and comment briefly on the other two cases.

Scaling equations (5) and (6) by $x \rightarrow X=\varepsilon x, t \rightarrow T=\varepsilon^{2} t$, we find

$$
\alpha=\pi / 2+O\left(\varepsilon^{2}\right), \quad \partial_{t} \beta=\nabla^{2} \beta+O\left(\varepsilon^{2}\right) .
$$

The equation for $\beta$ should be supplemented with the topological constraint

$$
\frac{1}{2 \pi} \oint \nabla \beta \cdot d l=n
$$

where the integration takes place along a contour surrounding the defect.

One could infer that the long scale behavior is dominated by diffusional relaxation of the azimuthal angle. The presence of a nonzero topological charge, however, implies that there must be a line cutting through the enclosed area where such description becomes inapplicable. The solution to our original problem need not be singular near this line. The reason is that the circle on $S^{2}$ described by (21) and (22) can be smoothly deformed to a point [7]. We should, though, restore there to the full short scale dynamics. Except for the vicinity of the disclinations, the $\beta$ field is governed by (21), coupled with the (yet unknown) vortex locations.

We now choose a system of coordinates attached to the defect. Fixing the $y$ axis in the direction of motion, (21) becomes

$$
\nabla^{2} \beta+w \partial_{Y} \beta=0
$$


where $w$ denotes the vortex speed. Note that (23) is invariant under the transformation

$$
\beta \rightarrow \beta+A X,
$$

where $A$ is some fixed constant. The phase gradient $A$ describes an external field (not to be confused with $H$ ) setting the defect into motion. Thus we assume

$$
\nabla \beta \sim A \hat{e}_{1}, \quad X^{2}+Y^{2} \rightarrow \infty .
$$

The goal is to find a relation between $w$ and $A$. This relation will be the law of motion for the defect.

A tricky method to solve equations like (23) with the condition (22) was proposed in [3]. The idea is to consider an approximate "conjugate" problem:

$$
\nabla^{2} \Phi+w \partial_{y} \Phi=2 \pi \delta(X) \delta(Y) .
$$

It is easy to check that $\Phi$ and $\beta$ are connected via

$$
n\left(\partial_{Y} \Phi+\Phi\right)=-\partial_{X} \beta, \quad n \partial_{X} \Phi=\partial_{Y} \beta .
$$

Now, the solution to (26) can be found by standard techniques:

$$
\Phi=\exp \left(-\frac{1}{2} w Y\right) K_{0}\left(\frac{1}{2} w R\right)=\exp \left(-\frac{1}{2} w R \sin \phi\right) K_{0}\left(\frac{1}{2} w R\right),
$$

where $R=\varepsilon r$ and $K_{j}$ denotes the modified Bessel function of order $j$. It is possible to integrate (27) to get $\beta$ in a closed form. For our purposes, however, it is enough to compute $\nabla \beta$. We obtain

$$
\begin{gathered}
\partial_{X} \beta=A+\frac{1}{2} n w \exp \left(-\frac{1}{2} w r \sin \phi\right)\left[K_{0}\left(\frac{1}{2} w R\right)-\sin \phi K_{1}\left(\frac{1}{2} w R\right),\right. \\
\partial_{Y} \beta=\frac{1}{2} n w \exp \left(-\frac{1}{2} w r \sin \phi\right) \cos \phi K_{1}\left(\frac{1}{2} w R\right) .
\end{gathered}
$$

We want to match (29) and (30) with the core expansion that will be computed in the next section. Therefore we expand these expressions in terms of the local variables:

$$
\begin{gathered}
\partial_{x} \beta=-\frac{n}{r} \sin \phi+\varepsilon A+\frac{1}{2} n \varepsilon w\left[-\ln \left(\frac{1}{4} \varepsilon w r\right)-C+\sin ^{2} \phi\right], \\
\partial_{y} \beta=\frac{n}{r} \cos \phi+\frac{1}{2} n \varepsilon w \cos \phi \sin \phi,
\end{gathered}
$$

where $C$ is the Euler constant.

In the same way, for both nematics with variable degree of orientation and smectics C, we get from (13) and (14)

$$
s=c+O\left(\varepsilon^{2}\right), \quad \partial_{t} \beta=\nabla^{2} \beta+O\left(\varepsilon^{2}\right) .
$$

The equation for the phase $\beta$ is considered with the conditions (22) and (25). To avoid the singularity implied by $(22)$, the field $s(x, t)$ undergoes a sharp transition from its equilibrium value $s=c$ to the value $s=0$ at the defect center. Except for the core itself, we get again (29)-(32) for $\beta$. In the case of smectics $C$, recalling the definition of $s$, we see the limit at $r \rightarrow 0$ is precisely the "escape into the third dimension" phenomenon [7]. Far from the disclination, $\beta$ is given by (29)-(32). 
4. The structure of the core. We again consider the case of nematics in external fields in detail. Returning the original $(x, t)$ coordinates, we write $(5)$ and (6) in the comoving frame:

$$
\begin{gathered}
\varepsilon w \partial_{y} \alpha+\nabla^{2} \alpha+\frac{1}{2}\left(1-|\nabla \beta|^{2}\right) \sin 2 \alpha=0, \\
\varepsilon w \partial_{y} \alpha+\nabla^{2} \beta+2 \cot \alpha \nabla \alpha \cdot \nabla \beta=0 .
\end{gathered}
$$

There are several ways to proceed [13]. We choose the more detailed matching procedure suggested in [11] since it reveals the precise nature of the core solution. For this purpose let

$$
\alpha=\alpha_{0}+\varepsilon w \psi(r) \sin \phi, \quad \beta=n \phi+\varepsilon w \chi(r) \cos \phi,
$$

where $\alpha_{0}$ is the function defined in (8). The first-order equations read

$$
\begin{gathered}
\psi^{\prime \prime}+r^{-1} \psi^{\prime}+\left[\left(1-n^{2} r^{-2}\right) \cos 2 \alpha_{0}+r^{-2}\right] \psi+n r^{-2} \chi \sin 2 \alpha_{0}+\alpha_{0}^{\prime}=0, \\
\chi^{\prime \prime}+r^{-1} \chi^{\prime}-r^{-2} \chi+2 \cot \alpha_{0}\left(\alpha_{0}^{\prime} \chi^{\prime}+n r^{-2} \psi\right)+n r^{-1}=0 .
\end{gathered}
$$

Equations (37), (38) are solved with the initial conditions

$$
\psi(0)=\psi^{\prime}(0)=\chi(0)=0
$$

that are required to remove the singularity at $r=0$.

An additional condition for $\chi$ is actually forced by the solvability condition for the system (37)-(38). To see this, we multiply (37) by $\alpha_{0}^{\prime}$ and (38) by $n r^{-1} \sin ^{2} \alpha_{0}$. Adding the two modified equations and integrating over a large disc of radius $\rho=$ $O\left(\varepsilon^{-1 / 2}\right)$ we get the following relation:

$$
\int_{0}^{\rho}\left(r\left(\alpha_{0}^{\prime}\right)^{2}+n^{2} r^{-1} \sin ^{2} \alpha_{0}\right) d r=\left[\alpha_{0}^{\prime \prime} \psi-\alpha_{0}^{\prime} \psi-n \sin ^{2} \alpha_{0}\left(r^{-1} \chi+\chi^{\prime}\right)\right]_{r=\rho} .
$$

Since $\alpha_{0}(r)=1-O(\exp (-r))$ as $r \rightarrow \infty$, the left-hand side of (40) can be expressed as $n^{2} \ln (\rho / L)$ where $L(n)$ is a numerical factor that can be easily computed from $\alpha_{0}$. For instance, $L(1)=0.344477$. We now turn our attention to the right-hand side of (40). The asymptotic behavior of $\chi$ for large values of $r$ can be read from (38):

$$
\chi=n\left(b r-\frac{1}{2} r \ln r\right)+O\left(r^{-1}\right) .
$$

It is easy to check now that (40) yields

$$
b(n)=\frac{1}{2}\left(\frac{1}{2}+\ln L(n)\right) .
$$

In particular we get $b(1)=-0.282864$. It follows that if one is only interested in matching the core expansion to the far field one, there is no need to actually solve for $\chi$ and $\psi$ for the entire range of $r$.

We can compare now the inner limit of the far field expansion (31), (32) with the outer limit of the core expansion $(36)-(41)$ to obtain the relation between the velocity of the disclination and the external field:

$$
\frac{2}{n} A=w \ln \left(\frac{\varepsilon w}{w_{0}}\right)
$$


where the constant $w_{0}$ is given by

$$
w_{0}(n)=4 \exp (1-C-2 b(n)) .
$$

For both smectics $\mathrm{C}$ and nematics with variable degree of orientation, the matching is carried out in the same way, yielding an identical mobility law, the only difference being in the numerical value of the constant $b$ or $w_{0}$. The analysis is very similar to the one carried out in [11]. Therefore we sketch the results without spelling out the details. We shall also set for simplicity $k=1$.

In the comoving frame, the terms $\partial_{t} s$ and $\partial_{t} \beta$ are replaced by $\varepsilon w \partial_{y} s$ and $\varepsilon w \partial_{y} \beta$, respectively. We then expand

$$
s=s_{0}(r)+\varepsilon w \chi(r) \sin \phi, \quad \beta=n \phi+\varepsilon w \psi(r) \cos \phi .
$$

The first-order equations for $\chi$ and $\psi$ are

$$
\begin{gathered}
\psi^{\prime \prime}+r^{-1} \psi^{\prime}-2 n r^{-2} \psi-V^{\prime}\left(s_{0}\right) \psi+2 n r^{-2} s_{0} \chi+s_{0}^{\prime}=0 \\
\chi^{\prime \prime}+r^{-1} \chi^{\prime}-n r^{-2} \chi+2 s_{0}^{-1}\left(s_{0}^{\prime} \chi^{\prime}+n r^{-2} \psi\right)+r^{-1}=0 .
\end{gathered}
$$

From (46) and (47) it is easy to infer

$$
\begin{gathered}
\psi=O\left(r^{-2}\right), \quad r \rightarrow \infty, \\
\chi=-\frac{1}{2} r^{-1} \ln ^{2} r+(2 b-1) r^{-1} \ln r+O\left(r^{-1}\right), \quad r \rightarrow \infty .
\end{gathered}
$$

The constant $b(n)$ can be computed directly [11] by solving equation (47). Alternatively, it can be found [12] from the numerical solution of (16) by a procedure analogous to (40). In the important case of $n=1$ one gets $b(1)=0.309$. Matching the inner and outer expansions, we get again the law of motion (43)-(44), with the appropriate value of $b(n)$.

5. Discussion. The above results can be seen as ingredients of the "particles+field" approximation for nonlinear ordered media. All the cases considered above describe an essentially two-dimensional (in the physical space) setting with two-dimensional order parameter space and the residual symmetry group of the minimal stratum is $U(1)$. The full field equations are nonlinear; however, they reduce to a linear heat equation for the "phase" $\beta$ far from the core regions of the defects. The defects can be seen as sources of the phase field, according to the circulation condition (22). In their turn, the defects move, according to the mobility relationship (43). Their motion is induced by the action of the phase gradient $A$ due to all other defects (and possibly other field components) at the core location. One can say therefore that the interaction of the defects is mediated by the phase field. Like particles, defects carry a conserved topological number (charge). Their equations of motion are not contained in the far field (phase) equations, and had to be deduced from the analysis of the full nonlinear system.

We see from (43) that to leading order in $\varepsilon$ the velocity is given by

$$
w=(\ln \varepsilon)^{-1} 2 A / n \text {. }
$$


From the analysis of the far field we see that the influence of a vortex at $r_{2}$ with charge $n_{2}$ on a vortex at $r_{1}$ of charge $n_{1}$ is given (to leading order) by

$$
n_{2} \nabla \phi\left(r_{2}-r_{1}\right) \text {. }
$$

To conform with our coordinates convention (cf. (23)), we set the vortex 1 at the center, with vortex 2 along the $y$ axis. Thus

$$
A=-\frac{n_{2}}{\left|r_{2}-r_{1}\right|}, \quad w=-(\ln \varepsilon)^{-1} \frac{2 n_{2}}{n_{1}\left|r_{2}-r_{1}\right|} .
$$

Thus defects with like charges repel while those oppositely charged attract each other. The interaction is, however, more complex than in classical field theories, like electromagnetism, and is more akin to hydrodynamical interaction, as it depends on the velocities as well as on positions of defects. The mobility relationships are, of course, applicable only when defects are sufficiently separated. When the density of defects is too large, the entire "particle+field" approximation breaks down.

The problem of mobility of point defects in two-dimensional ordered media has been studied in the past largely in the context of dynamics of nonequilibrium patterns [15]. For some time, the influence of the size of the system on the mobility was misunderstood. The confusion stemmed, in essence, from the logarithmic divergency of the energy of a single quiescent defect with the size of the system. The defect velocity was presented as the ratio of a thermodynamic driving force ("Peach-Köhler force," related to the external phase gradient) to a "dissipative integral" akin to (40) but evaluated over the entire region rather than over a disk with an $O\left(\varepsilon^{-1 / 2}\right)$ radius. The logarithmic divergency of this integral appeared to imply the logarithmic decrease of the mobility with the size of the system. The same conclusion has been stated recently in the context of mobility of defects in smectics $C$ [14].

This conclusion is flawed because the far field solution depends in a singular way on the defect velocity, so that the dissipative integral evaluated in the comoving frame converges even when the velocity is arbitrarily small [1]. The problem of divergencies does not arise in the above derivation of the defect mobility, based on the matching of solutions in inner (in the vicinity of the defect) and outer (far field) regions. The dependence on the size of the system is a subtle issue. It can be related to the influence of the shape of a planar region on the Green's function of the heat equation (21) or to the action of images of defects. The far field at any given moment depends upon the whole history of motion of defects. Therefore the evolution of the system has to be followed by solving the far field (phase) equation simultaneously with the equations of motion of the defects.

Acknowledgment. This work was supported by the U.S.-Israel Binational Science Foundation.

\section{REFERENCES}

[1] E. Bodenschatz, W. Pesch, and L. Kramer, Structure and dynamics of dislocations in anisotropic pattern-forming systems, Physica D 32, 135-145 (1988)

[2] H. Brezis, J-M. Coron, and E. Lieb, Harmonic maps with defects, Comm. Math. Phys. 107, 649705 (1986) 
[3] E. Dubois-Violette, E. Guazelli, and J. Prost, Dislocation motion in layered structures, Phil. Mag. A48, 727-747 (1983)

[4] J. Ericksen, Liquid crystals with variable degree of orientiation, IMA Preprint No. 559 (1989)

[5] P. G. de Gennes, The Physics of Liquid Crystals, Clarendon Press, Oxford, 1974

[6] R. Hardt, D. Kinderlehrer, and M. Luskin, Remarks about the mathematical theory of liquid crystals, Lecture Notes in Math., vol. 1340, Springer-Verlag, Berlin and New York, 1988, pp. 123-138

[7] M. Kleman, Points, lines and walls, John Wiley, New York, 1983

[8] _ Defects in liquid crystals, Rep. Progr. Phys. 52, 555-564 (1989)

[9] N. D. Mermin, The topological theory of defects in ordered media, Rev. Modern Phys. 51, 591-648 (1979)

[10] J. Neu, Vortices in complex scalar fields, Physica D 43, 385-406 (1990)

[11] L. Pismen and J. D. Rodriguez, Mobility of singularities in the dissipative Ginzburg-Landau equation, Phys. Rev. A 42, 2471-2474 (1990)

[12] L. Pismen and J. Rubinstein, Motion of vortex lines in the Ginzburg-Landau model, Physica D 47, 353-360 (1991)

[13] _ Dynamics of defects, preprint in Nematics: Mathematical and Physical Aspects, J. M. Coron et al. (eds.), Kluwer, 1991

[14] H. Pleiner, Dynamics of a disclination point in Smectic- $C$ liquid crystals, Phys. Rev. A 37, 39863992 (1988)

[15] Y. Pomeau, S. Zaleski, and P. Manneville, Disclination motion in cellular structures, Phys. Rev. A 27, 2710-2726 (1983)

[16] J. Rubinstein, Self-induced motion of line defects, Quart. Appl. Math. 49, 1-9 (1991) 